\title{
BMJ Open Evaluation of the efficacy of 3D total- body photography with sequential digital dermoscopy in a high-risk melanoma cohort: protocol for a randomised controlled trial
}

\author{
Clare Amy Primiero (D) , ${ }^{1}$ Aideen M Mclnerney-Leo, ${ }^{1}$ Brigid Betz-Stablein, ${ }^{1}$ \\ David C Whiteman, ${ }^{2}$ Louisa Gordon, ${ }^{3,4,5}$ Liam Caffery, ${ }^{1,6,7}$ Joanne F Aitken, ${ }^{5,8,9}$ \\ Elizabeth Eakin, ${ }^{10}$ Sonya Osborne, ${ }^{11}$ Len Gray, ${ }^{6}$ B Mark Smithers, ${ }^{10,12}$ \\ Monika Janda, ${ }^{1,6}$ H Peter Soyer, ${ }^{1,7}$ Anna Finnane ${ }^{5}$
}

To cite: Primiero CA, McInerney-Leo AM, BetzStablein B, et al. Evaluation of the efficacy of 3D total-body photography with sequential digital dermoscopy in a high-risk melanoma cohort: protocol for a randomised controlled trial. BMJ Open 2019;9:e032969. doi:10.1136/ bmjopen-2019-032969

- Prepublication history for this paper is available online. To view these files, please visit the journal online (http://dx.doi. org/10.1136/bmjopen-2019032969).

Received 15 July 2019 Revised 10 September 2019 Accepted 07 0ctober 2019

Check for updates

(C) Author(s) (or their employer(s)) 2019. Re-use permitted under CC BY-NC. No commercial re-use. See rights and permissions. Published by BMJ.

For numbered affiliations see end of article.

Correspondence to Dr H Peter Soyer; p.soyer@uq.edu.au

\section{ABSTRACT}

Introduction Melanoma is Australia's fourth most common cancer. Early detection is fundamental in maximising health outcomes and minimising treatment costs. To date, population-based screening programmes have not been justified in health economic studies. However, a skin surveillance approach targeting high-risk individuals could improve the cost-benefit ratio. Methods and analysis This paper describes a 2year longitudinal randomised controlled trial (RCT) to compare routine clinical care (control) with an intensive skin surveillance programme (intervention) consisting of novel three-dimensional (3D) total-body photography (TBP), sequential digital dermoscopy and melanomarisk stratification, in a high-risk melanoma cohort. Primary outcomes will evaluate clinical, economic and consumer impact of the intervention. Clinical outcomes will evaluate differences in the rate of lesion excisions/ biopsies per person, benign to malignant ratio for excisions and thickness of melanomas diagnosed. A health economic analysis using government data repositories will capture healthcare utilisation and costs relating to skin surveillance. Consumer questionnaires will examine intervention acceptability, the psychological impact, and attitudes towards melanoma risk and sun protective behaviour. Secondary outcomes include the development of a holistic risk algorithm incorporating clinical, phenotypic and genetic factors to facilitate the identification of those most likely to benefit from this surveillance approach. Furthermore, the feasibility of integrating the intervention with teledermatology to enhance specialist care in remote locations will be evaluated. This will be the first RCT to compare a targeted surveillance programme utilising new 3D TBP technology against current routine clinical care for individuals at high risk of melanoma.

Ethics and dissemination This study has received Human Research Ethics Committee (HREC) approval from both Metro South Health HREC (HREC/17/ QPAH/816) and The University of Queensland HREC (2018000074).

\section{Strengths and limitations of this study}

- The first randomised controlled trial to compare three-dimensional (3D) total-body photography (TBP) to standard-of-care for people who are at high risk of melanoma.

- Large sample size, recruited from research volunteer registry and dermatologist referrals with a projected high retention rate.

- Collection of longitudinal data from government health repositories will allow a relatively complete, rich data set on healthcare use and costs relevant to melanoma risk.

- Study will evaluate feasibility of using 3D TBP for telediagnosis.

- Limited outcomes assessment, with absence of gold-standard mortality as an endpoint.

Trial registration number ANZCTR12618000267257; Pre-results.

\section{INTRODUCTION}

Australia, with a population of only 25 million residents, has one of the highest rates of cutaneous melanoma incidence and mortality in the world, with over 13000 new cases diagnosed in 2016, and over 1700 deaths. ${ }^{1}$ In 2014, the costs of advanced melanoma were estimated to be \$A422 million nationally of which $39 \%$ was attributable to direct healthcare costs. ${ }^{2}$ Early detection of melanoma is preferred because more advanced stage melanomas have poorer patient outcomes and are costly to manage. ${ }^{3-5}$ While populationbased screening is not warranted, identifying people at high risk of melanoma could enable targeted screening, and be the most effective way of improving early detection. Extensive 
research efforts have been undertaken to improve strategies for identifying and following those at greatest risk. ${ }^{6}$ However, there is currently no consensus on the best risk assessment or surveillance strategies. As a result, screening recommendations vary and are inconsistently applied. ${ }^{7}$

Current approaches for identifying and screening those at greatest risk are imperfect. ${ }^{8}$ Risk prediction tools have been developed, and involve weighting a subset of risk factors including phenotypic features, personal history and, more rarely, genetic test results. The most important markers of individual melanoma risk include: $C D K N 2 \mathrm{~A}$ germline mutation, having $>100$ naevi, $>5$ atypical naevi, fair hair, eye and skin colour, a strong family history or a personal history of melanoma. ${ }^{6910}$ Within Australia, dermatologists typically adhere to The Cancer Council Australia's guidelines, that recommend high-risk individuals undergo clinical skin examinations every 6 months using total-body photography (TBP) in combination with sequential digital dermoscopy imaging (SDDI). ${ }^{11} 12$

TBP provides a comparative record of the skin surface assisting in identification of new lesions and to an extent, changes of existing naevi. Dermoscopy enables the visualisation of the surface morphology of pigmented skin lesions and reveals colours and structures that normally are not visible to the naked eye. Dermoscopy has consistently been shown to improve the diagnostic accuracy of melanomas, ${ }^{13}$ and when used across visits it is particularly useful for detection of incipient melanomas, which lack typical dermoscopy features. ${ }^{14}$ This 'two step' process involving both TBP and dermoscopy for skin surveillance was initially described in $2002^{15}$ and has been repeatedly shown to be associated with a lower benign to malignant excision ratio and decreased Breslow thickness of subsequently diagnosed melanomas. ${ }^{4} 131617$ More recently, surveillance of clinical patients at high risk of melanoma has demonstrated efficacy and cost-effectiveness. A large 10-year, retrospective review of a high-risk clinic in Sydney, Australia reported that patients were diagnosed with thinner melanomas, and underwent fewer excision compared with standard care. ${ }^{4}$ A review of previous studies exploring targeted screening for melanoma using visual skin inspection reported mixed findings for costeffectiveness depending on the baseline level of risk for the targeted population. ${ }^{18}$

Surveillance strategies have evolved significantly over the past decade. One of the most promising approaches involves automated three-dimensional (3D) imaging of subjects, allowing objective documentation of all existing lesions and monitoring changes over time. The VECTRA WB360 system described previously ${ }^{19} 20$ allows fast 3D TBP and construction of a patient avatar, along with integrated dermoscopy. Consumer feedback indicates high acceptability and confidence in the technology for skin monitoring, and importantly, whole body imaging may reduce melanoma-related anxiety. ${ }^{19}$ The advances in total-body imaging in recent years were unprecedented, and now enable high resolution 3D imaging in minutes. The impact of these changes on health economic models of high-risk screening warrants investigation. It is the objective of the current study to examine the use of 3D imaging technology for targeted surveillance for individuals at high risk to melanoma.

The geographical distribution of the population in Australia creates additional challenges to equitable health service delivery. ${ }^{21}$ Melanoma patients in rural areas of Australia are often disadvantaged with inequitable access to dermatological care and are reported to suffer $20 \%$ increased melanoma-related mortality compared with urban areas. ${ }^{21}$ Already now, rural physicians are using teledermatology to obtain second opinions on suspicious lesions from specialist dermatologists. ${ }^{22}$ Incorporating 3D TBP into a teledermatology service could facilitate remote full body skin examination by teledermatologists reflecting the service level that urban patients can access. The current study will evaluate the feasibility of using a telehealth network for transmission of 3D TBP-SDDI images, and the concordance of diagnostic decisions between in-person skin examinations and remote teledermatologist review.

This study will recruit individuals that are at high risk of developing cutaneous melanoma to participate in a randomised trial comparing combined TBP and SDDI surveillance approach (3D TBP-SDDI), with routine clinical care. TBP and SDDI reportedly improve earlier detection rates ${ }^{13}{ }^{23}$; however, the effect of $3 \mathrm{D}$ TBP is unknown. The feasibility of extending its use through a telehealth network will be explored. An evaluation of efficacy, costs and consumer acceptability of the technology will determine long-term sustainability. Furthermore, a standardised, holistic approach to risk stratification for melanoma will be developed, optimising the identification of those who would most benefit from this high-risk surveillance programme. This study is an integral step in guiding change in the way high-risk individuals may be managed in Australia.

\section{OBJECTIVES}

\section{Primary objectives}

- Compare clinical outcomes of the 3D TBP-SDDI approach with routine clinical care, including numbers of excisions or biopsies and histopathological findings.

- Compare health economic outcomes of the 3D TBPSDDI approach with routine clinical care.

- Evaluate consumer acceptance of the intervention, psychological well-being, health behaviour and beliefs regarding sun protection and melanoma.

\section{Secondary objectives}

- Assess feasibility of telehealth to deliver remotely captured 3D TBP-SDDI for teledermatologist review.

- Evaluate the degree of concordance between teledermatologist and in-person examination in terms of clinical assessment and management decisions. 
- Identify rare and deleterious gene variants associated with melanoma risk.

- Refine a risk stratification model that combines medical history, family history, phenotypic risk factors and genetic results to produce a melanoma-risk score.

\section{METHODS AND ANALYSIS}

\section{Study design and setting}

A two-arm, single-site, parallel randomised controlled trial (RCT) will recruit 330 participants, with a 50:50 allocation ratio between intervention and control groups. Study visits commenced in April 2018, and are expected to be completed by August 2021. The study site will be the Clinical Research Facility of the Translational Research Institute at the Princess Alexandra Hospital, Brisbane. The majority of study participants reside in South-East Queensland, Australia.

\section{Participant and public involvement}

Prior to applying for funding, we have conducted clinical research recruiting both average risk and high-risk members of the public regarding skin surveillance since 2010, and have used questionnaires for participant feedback regarding skin cancer prevention which has contributed to the current study design. Since 2016, we have held biannual consumer forums to inform the public and our study participants of our research progress, and to give consumers the opportunities to discuss their priorities and concerns regarding skin cancer prevention with our group.

\section{Eligibility criteria}

Individuals that are at high risk of developing a primary or subsequent primary melanoma will be invited to participate. High risk will be defined as having one of the following:

- At least one melanoma (including in situ) diagnosed before the age of 40 years.

- Two or more melanomas (including in situ) diagnosed before the age of 65 years.

- A strong family history (2+ first-degree relatives) and/ or known pathogenic genetic mutation and/or a diagnosis of dysplastic naevus syndrome.

\section{Recruitment}

Participants will be recruited from a registry of research volunteers with the University of Queensland, Dermatology Research Centre, and by referrals from dermatologists and medical practitioners from South-East Queensland, over a 12-18-month period. Potential participants will be emailed a short description of the study and a copy of the participant information and consent form, followed by a phone call approximately 2 weeks thereafter.

\section{Randomisation and blinding}

Once consented at the initial baseline visit, participants will be randomised to either the intervention or control group with simple random sampling using the randomisation function in Research Electronic Data Capture
(REDCap). REDCap is a secure, online study database software developed by Vanderbilt University, and administrated by The Queensland Clinical Trials and Biostatistics Centre at the School of Public Health, The University of Queensland. Simple randomisation method is selected as it is an agnostic approach which is straightforward to implement. It is acknowledged that this approach is vulnerable to random sampling errors; however, we will account for this in our secondary analysis. An online random number generator ${ }^{24}$ will create an allocation sequence table which will be uploaded to REDCap. Trial staff conducting patient visits will be blinded to the next allocation sequence. Due to the nature of the intervention once participants are randomised, allocation is unblinded.

To address the teledermatology objectives, a subset of participants will be evaluated two ways. First by 3D TBPSDDI and a face-to-face dermatologist, and second their images alone will be independently evaluated by another dermatologist.

\section{Intervention}

Participants randomised to the intervention group will receive clinical skin examinations, every 6 months for 2 years, supported by the 3D TBP imaging system (VECTRA WB360, serial number WB00009, Canfield Scientific, Parsippany, New Jersey, USA). The VECTRA imaging system consists of a framework of 92 cameras, which simultaneously capture images of the participant holding one anatomical pose, to construct a 3D avatar. An attached dermoscopic camera (EOS Rebel T6i) enables imaging of individual naevi including anatomical localisation on the 3D avatar. Clinical skin examinations are performed at the time of imaging by dermatologicallytrained medical practitioners, and images are reviewed and discussed fortnightly with an accredited dermatologist, simulating a teledermatology consultation to assess 3D TBP avatars and corresponding dermoscopic images. Suspicious lesions will be discussed with the study participant by phone, and subsequently referred to their treating physician. Participants will be asked to continue attending their regular skin examination appointments.

\section{Control}

Participants randomised to the control group will be asked to continue attending their regular skin examination appointments (which may include two-dimensional (2D) TBP), and to complete six monthly questionnaires (as described in table 1 and figure 1). At the end of the study, all control participants will be offered a clinical skin examination including 3D TBP imaging.

\section{Participant timeline}

Participants in both groups will be evaluated over a 2-year period from baseline, with the intervention group attending visits at baseline, 6, 12, 18 and 24 months. The control group will attend the clinic in person at baseline and at 24 months, and will complete an online 
Table 1 Primary outcomes and methods assessments

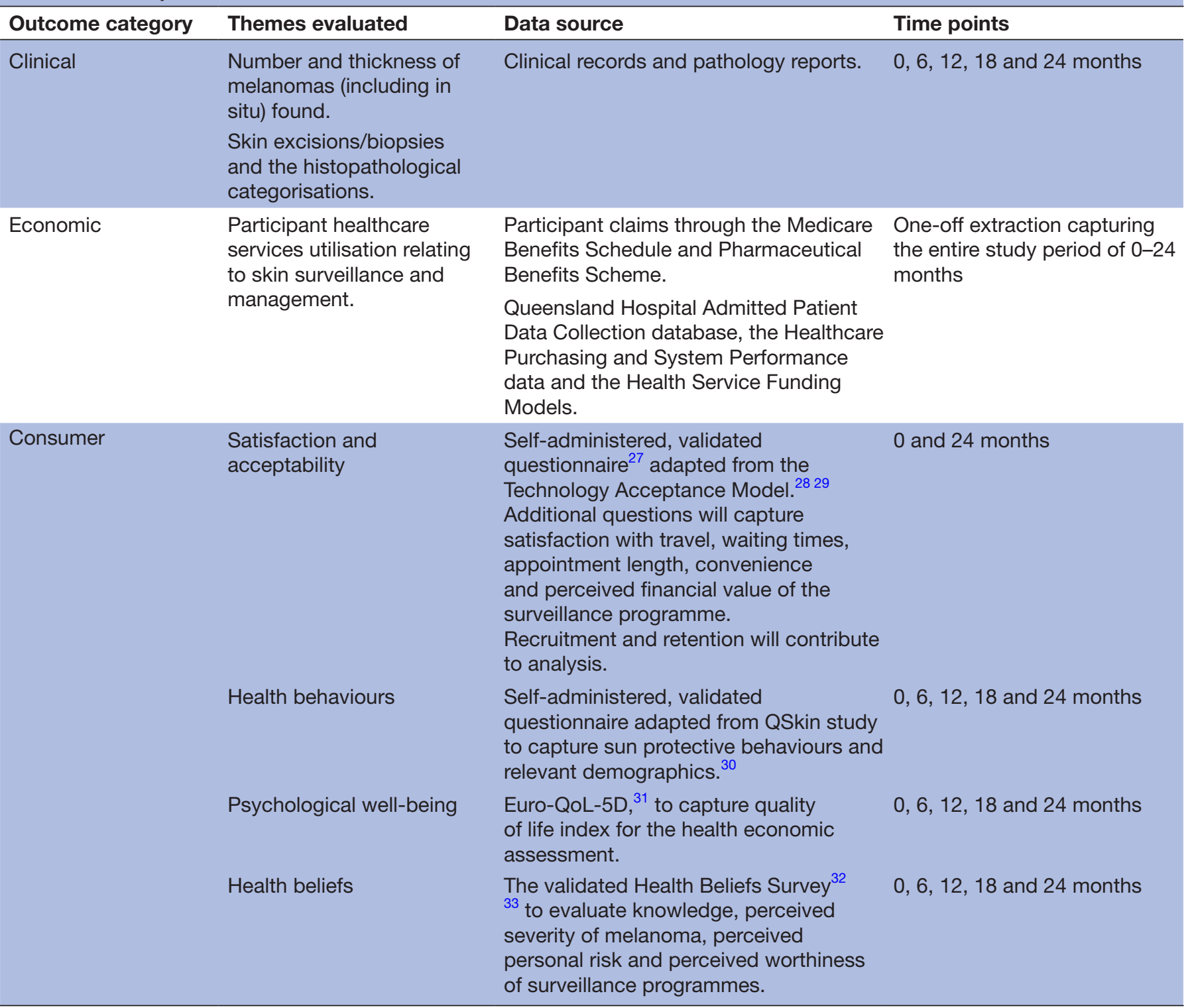

questionnaire at months 6,12 and 18. Refer to figure 1 for an overview of participant timeline and assessments.

\section{Primary outcomes}

The primary outcome for this study is the number of excisions. The other main outcomes are defined in three categories, including clinical, economic and consumer outcomes. The assessments of these outcomes are described in table 1.

\section{Secondary outcomes}

There are three secondary outcomes: the feasibility of viewing 3D TBP-SDDI images remotely using the telehealth network; the efficacy of performing skin examinations using 3D TBP-SDDI images using telehealth services and lastly the identification of genetic mutations and their utility in melanoma-risk stratification. The assessments of these outcomes are described in table 2.

\section{Data collection and management}

Baseline questionnaire and clinical data will be entered into the REDCap database. 3D TBP-SDDI images will be captured using the VECTRA imaging system and integrated software. Pathology reports will be requested from the Queensland Cancer Registry and medical records. One-off extractions of claims and health service data will occur at the end of follow-up from the Medicare Benefits Schedule (MBS) and Pharmaceutical Benefits Scheme (PBS), the Queensland Hospital Admitted Patient Data Collection database, the Healthcare Purchasing and System Performance data, and the Hospital and Health Service Funding Models. By linking data, the whole journey of healthcare service contacts and patient skin cancer outcomes will be captured, to allow an estimate of related costs of skin cancers. Costs will be analysed from the health provider (government) perspective. 


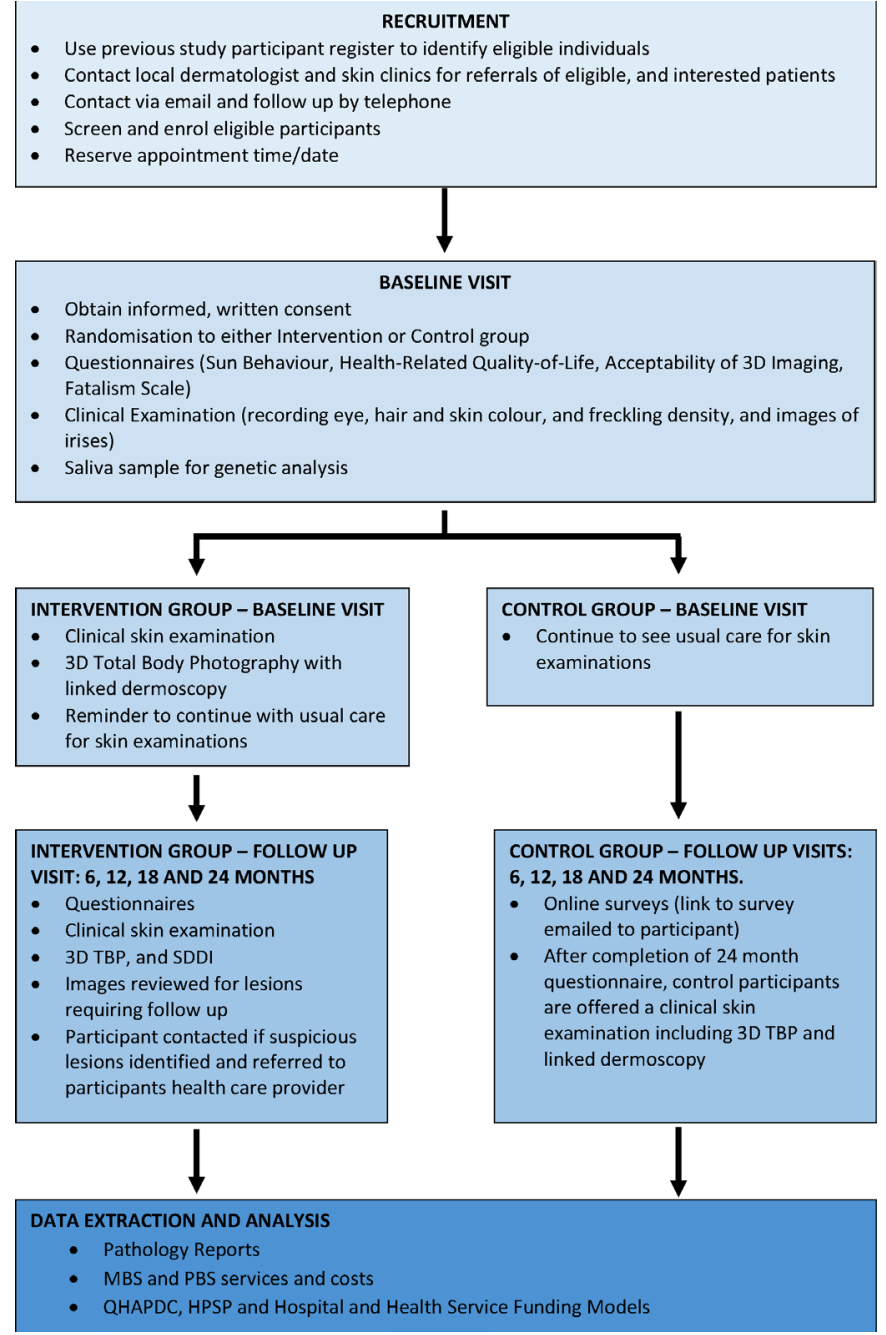

Figure 1 Overview of participant timeline and assessments.

Intervention resources will be compiled and monitored by the project manager.

Saliva samples and subsequent sequencing results will be coded and linked using a re-identifiable 'study ID'. Data linking identifying information and study ID will be stored in a password-controlled database, on a secure server, accessible to a limited subset of the study team to ensure privacy.

The REDCap management system, the VECTRA images and all remaining electronic data will be stored under The University of Queensland Research Data Management system on a secured network. Identifiers are removed from all participant data and replaced with a unique study ID to further protect privacy. Regular quality assurance checks of image data and REDCap entries will be conducted.

\section{Data monitoring}

The study team determined that an independent data monitoring committee was not required as the risk to study participants was low, mainly relating to privacy and the possibility of unnecessary excision or biopsies. Privacy risks are mitigated as discussed above. An 'issues register' will be kept to record technical problems that occur during trial visits. Interviews with key stakeholders will identify problematic procedures. Any concerns with data quality or issues recorded will be discussed at regular team meetings. The study team will perform regular data monitoring and quality assurance tasks internally, and any protocol deviations or adverse events will be reported to the ethics committee.

\section{STATISTICAL METHODS \\ Sample size}

The study aims to recruit 330 high-risk participants over a 12-18-month period, to be powered to compare excision rates between groups. This sample size is based on previously reported difference in excision rates in a high-risk sample, between those monitored by TBP (mean $=0.81$, $\mathrm{SD}=0.75$ ) and a standard care group (mean $=2.55$, $\mathrm{SD}=2.01) .{ }^{4}$ The definition of high risk is broader in this study, and approximately one-third of participants are likely to be already monitored using TBP, therefore the difference between routine clinical care and monitoring with 3D TBP is likely to be smaller. Given this, the study was powered to observe a $50 \%$ smaller difference in excision rates than observed previously, ${ }^{4}$ including an increase in SD of 50\% within each group (mean intervention 0.81 $(\mathrm{SD}=1.13)$, mean control $1.68(\mathrm{SD}=3.02))$. Given these estimates, with a power of $90 \%$ and a significance level of $5 \%, 153$ participants will be required for each arm of the trial. Allowing for participant withdrawal, we will aim to recruit a total 330 participants.

\section{Baseline demographic}

Descriptive statistics will be used to summarise demographic and clinical characteristics for both the control and intervention groups. $\chi^{2}$ tests will be used to estimate difference in proportions of categorical variables between the two groups, and t-tests will be used for continuous variables. Non-parametric equivalents will be used if the assumptions of the parametric tests are violated. Results will be considered statistically significant if $\mathrm{p}<0.05$.

\section{Clinical outcomes}

A non-parametric Mann-Whitney test will be used to assess the clinical primary outcome to test if there is a difference in mean annual rate of lesion excisions/biopsies between the intervention and control groups, given the primary outcome is based on counts and therefore unlikely to follow a normal distribution. The primary outcome will be analysed as intention to treat, with a per protocol analysis as a secondary outcome. This outcome will also be re-evaluated on a subset excluding those from both the intervention and control groups who are receiving 2D TBP. The benign to malignant ratio for excisions of pigmented lesions and non-melanoma skin cancers will be calculated for both groups. $\chi^{2}$ test or fisher's exact test (as appropriate) will be used to compare the difference in proportions of pathology confirmed melanoma, 
Table 2 Secondary outcomes and methods assessments

\begin{tabular}{|c|c|c|c|}
\hline Outcome category & Factors evaluated & Data source & Time points \\
\hline $\begin{array}{l}\text { Feasibility of telehealth } \\
\text { approach }\end{array}$ & $\begin{array}{l}\text { Technical feasibility of } \\
\text { telehealth network for remote } \\
\text { dermatological review of } \\
\text { 3D TBP-SDDI images, and } \\
\text { interoperability with hospital } \\
\text { image repositories and } \\
\text { integrated electronic medical } \\
\text { records }\end{array}$ & $\begin{array}{l}\text { Investigate subsystems for image acquisition, } \\
\text { storage and display by measuring network } \\
\text { throughput (bandwidth) and latency between } \\
\text { subsystems. } \\
\text { Measure data volume and transmission time } \\
\text { per 3D TBP-SDDI examination. } \\
\text { Assess the compression ratio of transmitted } \\
\text { image files necessary to achieve adequate } \\
\text { functionality. } \\
\text { Evaluate success of transmission and } \\
\text { integrity of data. }\end{array}$ & 24 months \\
\hline $\begin{array}{l}\text { Accuracy of telehealth skin } \\
\text { examinations }\end{array}$ & $\begin{array}{l}\text { Safety and accuracy of } \\
\text { teledermatology review of 3D } \\
\text { TBP-SDDI images }\end{array}$ & $\begin{array}{l}\text { Review the concordance between provisional } \\
\text { diagnosis and clinical management decisions } \\
\text { of the teledermatologist to the gold standard } \\
\text { of in-person dermatological assessment. } \\
\text { Assess comparative diagnostic accuracy } \\
\text { between in-person clinical diagnosis, } \\
\text { teledermatological diagnosis and } \\
\text { histopathological diagnoses. }\end{array}$ & 0-24 months \\
\hline \multirow[t]{2}{*}{$\begin{array}{l}\text { Melanoma-risk stratification } \\
\text { in a high-risk population }\end{array}$} & \multirow[t]{2}{*}{ Genetic results } & $\begin{array}{l}\text { Saliva samples collected using Oragene } \\
\text { DNA self-collection kit. Methods for sample } \\
\text { processing described previously. }\end{array}$ & Baseline \\
\hline & & $\begin{array}{l}\text { Whole exome sequencing or Sanger } \\
\text { sequencing used to identify rare, pathogenic, } \\
\text { germline variants in known melanoma genes. } \\
\text { Common variants associated with melanoma } \\
\text { risk will be genotyped using Illumina } \\
\text { CoreExomev24 chip array. }\end{array}$ & $\begin{array}{l}\text { 12-24 } \\
\text { months }\end{array}$ \\
\hline
\end{tabular}

3D, three dimensional; SDDI, sequential digital dermoscopy imaging; TBP, total-body photography.

melanoma in situ, basal cell carcinoma and squamous cell carcinoma diagnosed between the two groups with diagnosis. Logistic regression analyses will be used to investigate the relationship between baseline demographic and phenotypic information, and past melanoma history. A subgroup analysis of participants diagnosed with melanoma excluding in situ and melanoma including in situ will investigate the differences in staging, Breslow thickness and body site and other parameters of interest, between the two groups, using linear, logistic and generalised regression models as appropriate. As above, results will be considered statistically significant if $p<0.05$. As 
primary outcome data will be collected through Medicare information consented to at baseline, there will be no or very minimal missing data. Therefore assuming the data are missing at random, we will remove participants with no outcome data from that analysis.

\section{Economic outcomes}

The economic analysis will assess the resource and cost differences between arms rather than a full economic evaluation due to the relatively short follow-up and small sample with which to detect health outcomes such as skin cancers. Data from Medicare and Queensland Health sources will be linked and aggregated for each patient covering the surveillance period of the study. Skin cancer related resource use will be identified and coded according to ICD-10 (International Classification of Diseases), procedure and MBS/PBS items. Cost data are typically skewed so generalised linear models will be used with a gamma family and log link (if appropriate) to assess differences between the intervention and control groups. Non-parametric bootstrapping methods will also be applied for verification of differences in costs between groups. Subgroup cost analyses of hospital versus outof-hospital, melanoma stage, age, phenotypes or other patient characteristics will be explored.

\section{Telehealth review outcomes}

The main telehealth outcome is the level of agreement in clinical decision between the teledermatologist and the dermatologist carrying out an in-person skin examination assessment. Four decision outcomes will be considered: No action (no suspicious lesion), follow-up in 3-6 months, excision of lesion(s) and treatment of lesion. Weighted Cohen's kappa coefficient will be calculated to measure agreement across the four categories using $\mathrm{R}$ package irr. ${ }^{25} \mathrm{~A}$ kappa between 0.6 and 0.79 will indicate substantial agreement, while a kappa of greater than 0.8 will indicate almost perfect agreement. ${ }^{26}$

\section{Melanoma-risk score development}

Multivariable logistic regression will be used to assess what combinations of genetic, phenotypic and demographic risk factors are associates with an increased odds of melanoma within a high-risk population. Variables with $\mathrm{p}<0.2$ in univariate regression will be included in the model, and backwards stepwise regression will be used, with variables in the model remaining statistically significant at the $5 \%$ level. Validation of the resulting risk stratification algorithms will be performed on larger collaborative cohorts.

\section{Consumer perspectives}

Data will be collected to determine the acceptability and feasibility of 3D imaging and any potential barriers and facilitators to implementation and adoption. This will include information related to convenience, comfort, and reasons for participant retention/loss and data on quality of life and fear of recurrence.

Questionnaire data will be prepared according to each scale's manual and standard procedures. ${ }^{27-33}$ Total and subscale scores will be computed and tested for normality. If normally distributed, parametric tests will be used, otherwise non-parametric analytic procedures will be used as described above to assess differences between the two groups in the consumer self-reported outcomes.

\section{ETHICS AND DISSEMINATION}

The protocol has been prepared in concordance with the Standard Protocol Items Recommendations for Interventional Trials (SPIRIT) statement. ${ }^{34}$ Following national guidelines, if genetic sequencing reveals a high penetrance pathogenic mutation, clinical genetic testing will be offered to the participant. A reimbursement of $\$ A 20$ per visit will be paid to participants to assist in covering travel and/or parking costs. Study results will be disseminated through peer-reviewed publications, conferences and non-peer reviewed media outlets.

\section{DISCUSSION}

This will be the first RCT to assess the feasibility, efficacy and cost-effectiveness of combining 3D TBP, with SDDI for clinical skin examinations of individuals at high risk to melanoma in Australia. Consumer perceptions of the technology and its clinical utility will also be assessed. This study will enable us to determine whether excision rates and stage of melanoma detection are affected by the inclusion of 3D TBP-SDDI in a surveillance protocol. Exploring the societal and personal costs of the intervention will be invaluable in determining the feasibility of incorporating this technology in routine clinical care for this high-risk cohort. The study will determine whether remote administration of 3D TBP, with SDDI combined with teledermatologist evaluation will affect clinical management. For greater public benefit in the longer term, it is critical to be able to accurately identify high-risk individuals who might benefit from this more intensive surveillance approach and, therefore, this study aims to develop a holistic risk stratification algorithm.

Australia currently has three national population-based screening programmes for early detection of breast, cervical and bowel cancers. However, there is no similar programme for skin cancer, despite melanoma being Australia's fourth most common cancer. ${ }^{10}$ The number needed to screen (NNS) in the Australian general public to save one life from melanoma has been estimated at $25000 .^{35}$ Therefore, population-based screening for melanoma may not be justified. However, by focusing on a high-risk population the probability of detecting a melanoma increases and the NNS decreases. Furthermore, the incorporation of TBP-SDDI enables a "watch and wait' approach which would reduce the number needed to treat based on the benign to malignant excision ratio, resulting in health cost savings. ${ }^{11}$ Criteria for an effective screening programme, outlined by the Australian Institute for Health and Welfare, ${ }^{36}$ stipulate that: the disease must be highly prevalent; the natural course of the disease 
is well understood including a recognisable latent or early symptomatic stage where disease can be detected; there is available treatment which is effective and well accepted; the disease must cause considerable costs, both fiscally and clinically; and lastly, the screening programme must be cost effective. With the criteria in mind, the current protocol is intended to build the evidence for a future targeted surveillance programme for melanoma detection in high-risk individuals in Australia, including solutions for geographical challenges. Economic evaluation of resource use and associated costs collected through linkage of clinical and administrative healthcare data sources will capture the whole journey of health service contacts and outcomes, to accurately estimate the related costs of screening and skin cancers within this high-risk population.

\section{CONCLUSION}

This protocol will provide evidence as to whether pursuing the incorporation of 3D TBP-SDDI into a surveillance programme for high-risk individuals can be cost effective and provide superior clinical outcomes over the current routine clinical care. Secondary outcomes will drive solutions in defining the population which would maximally benefit from this programme, and determine the acceptability of this surveillance method. Furthermore, the study will determine if the 3D TBP-SDDI technology is suitable for review through telehealth services, supporting solutions for outreach to remote regions of the country.

\section{Author affiliations}

${ }^{1}$ The University of Queensland Diamantina Institute, Dermatology Research Centre, The University of Queensland, Brisbane, Queensland, Australia

${ }^{2}$ Cancer Control Group, QIMR Berghofer Medical Research Institute, Brisbane, Queensland, Australia

${ }^{3}$ Population Health, QIMR Berghofer Medical Research Institute, Brisbane, Queensland, Australia

${ }^{4}$ School of Nursing, Queensland University of Technology, Brisbane, Queensland, Australia

${ }^{5}$ School of Public Health, The University of Queensland, Brisbane, Queensland, Australia

${ }^{6}$ Centre for Online Health, Centre for Health Services Research, The University of Queensland, Woolloongabba, Queensland, Australia

${ }^{7}$ Department of Dermatology, Princess Alexandra Hospital, Brisbane, Queensland, Australia

${ }^{8}$ Cancer Research Centre, Cancer Council Queensland, Brisbane, Queensland, Australia

${ }^{9}$ Institute for Resilient Religions, University of Southern Queensland, Springfield, Queensland, Australia

${ }^{10}$ Faculty of Medicine, The University of Queensland, Brisbane, Queensland, Australia

${ }^{11}$ School of Nursing and Midwifery, University of Southern Queensland, Toowoomba, Queensland, Australia

${ }^{12}$ Queensland Melanoma Project, Princess Alexandra Hospital, Brisbane, Queensland, Australia

Twitter Brigid Betz-Stablein @ClinicalOutlier, Louisa Gordon @louisagord and Liam Caffery @DrLiamCaffery

Contributors CAP, AMM-L, BB-S, DCW, LGo, LC, JFA, EE, SO, LGr, BMS, MJ, HPS and $A F$ were all involved in developing the study protocol. HPS, AF, MJ, BMS, LGr, S0, EE, JFA, LC, LGo and DCW worked together on the funding proposal. BB-S provided support for the development of the statistical analysis plan. All authors reviewed, edited and approved the final version.

Funding The research is funded by the National Health and Medical Research Council (NHMRC) partnership grant (APP1153046), Queensland Genomics, Queensland Government (M1215057), Princess Alexandra Research Foundation Translational Research Innovation Award, Brisbane Diamantina Health Partners Health System Improvement Ideas grant funded from Medical Research Future Fund Rapid Applied Research Translation Programme. CAP is funded by an Australian Government Research Training Programme Scholarship. AMM-L is funded via an NHMRC ECF APP1158111. MJ is funded by NHMRC TRIP Fellowship APP1151021. DCW is funded by an NHMRC Senior Principal Research Fellowship APP APP1155413. HPS is funded by an NHMRC Practitioner Fellowship APP1137127. The Translational Research Institute is supported by a grant from the Australian Government.

Competing interests HPS is a shareholder of MoleMap NZ Limited and e-derm consult $\mathrm{GmbH}$, and undertakes regular teledermatological reporting for both companies. HPS is a Medical Consultant for Canfield Scientific Inc., a Medical Advisor for First Derm, and has a Medical Advisory Board Appointment with MoleMap NZ Limited.

Patient consent for publication Not required.

Ethics approval This study has received Human Research Ethics Committee (HREC) approval from Metro South Health HREC (HREC/17/QPAH/816) and The University of Queensland HREC (2018000074).

Provenance and peer review Not commissioned; externally peer reviewed.

Open access This is an open access article distributed in accordance with the Creative Commons Attribution Non Commercial (CC BY-NC 4.0) license, which permits others to distribute, remix, adapt, build upon this work non-commercially, and license their derivative works on different terms, provided the original work is properly cited, appropriate credit is given, any changes made indicated, and the use is non-commercial. See: http://creativecommons.org/licenses/by-nc/4.0/.

ORCID iD

Clare Amy Primiero http://orcid.org/0000-0002-2944-0013

\section{REFERENCES}

1 (AlHW) AloHaW. Skin cancer in Australia Canberra: AlHW, 2016. Available: www.aihw.gov.au

$2 \mathrm{mpa}$. Advanced Melanoma - The real cost of Australia's national cancer: KPMG, 2014. Available: https://melanomapatients.org.au/ wp-content/uploads/2017/05/MPA_the_real_cost-1.pdf

3 Baade PD, Royston P, Youl PH, et al. Prognostic survival model for people diagnosed with invasive cutaneous melanoma. BMC Cancer 2015;15:27.

4 Watts CG, Cust AE, Menzies SW, et al. Cost-Effectiveness of skin surveillance through a specialized clinic for patients at high risk of melanoma. J Clin Oncol 2017;35:63-71.

5 Balch CM, Gershenwald JE, Soong S-J, et al. Final version of 2009 AJCC melanoma staging and classification. J Clin Oncol 2009;27:6199-206.

6 Vuong K, McGeechan K, Armstrong BK, et al. Risk prediction models for incident primary cutaneous melanoma: a systematic review. JAMA Dermatol 2014;150:434-44.

7 Olsen CM, Neale RE, Green AC, et al. Independent validation of six melanoma risk prediction models. $J$ Invest Dermatol 2015:135:1377-84

8 Bibbins-Domingo K, Grossman DC, Curry SJ, et al. Screening for skin cancer: US preventive services Task force recommendation statement. JAMA 2016;316:429-35.

9 Usher-Smith JA, Emery J, Kassianos AP, et al. Risk prediction models for melanoma: a systematic review. Cancer Epidemiol Biomarkers Prev 2014;23:1450-63.

10 Watts CG, Cust AE, Menzies SW, et al. Specialized surveillance for individuals at high risk for melanoma: a cost analysis of a high-risk clinic. JAMA Dermatol 2015;151:178-86.

11 Adler NR, Kelly JW, Guitera P, et al. Methods of melanoma detection and of skin monitoring for individuals at high risk of melanoma: new Australian clinical practice. Med J Aust 2019;210:41-7.

12 Mann G, Cust A, Damian D, et al. Clinical practice guidelines for the diagnosis and management of melanoma, 2018. Available: https:// wiki.cancer.org.au/australiawiki/index.php?oldid $=186052$

13 Salerni G, Carrera C, Lovatto L, et al. Benefits of total body photography and digital dermatoscopy ("two-step method of digital 
follow-up") in the early diagnosis of melanoma in patients at high risk for melanoma. J Am Acad Dermatol 2012;67:e17-27.

14 Kittler H, Guitera P, Riedl E, et al. Identification of clinically featureless incipient melanoma using sequential dermoscopy imaging. Arch Dermatol 2006;142:1113-9.

15 Malvehy J, Puig S. Follow-Up of melanocytic skin lesions with digital total-body photography and digital Dermoscopy: a two-step method. Clin Dermatol 2002;20:297-304

16 Moloney FJ, Guitera P, Coates E, et al. Detection of primary melanoma in individuals at extreme high risk: a prospective 5-year follow-up study. JAMA Dermatol 2014;150:819-27.

17 Halpern AC, Lieb JA. Early melanoma diagnosis: a success story that leaves room for improvement. Curr Opin Oncol 2007;19:109-15.

18 Gordon LG, Brynes J, Baade PD, et al. Cost-Effectiveness analysis of a skin awareness intervention for early detection of skin cancer targeting men older than 50 years. Value Health 2017;20:593-601.

19 Janda M, Soyer HP. Using advances in skin imaging technology and genomics for the early detection and prevention of melanoma. Dermatology 2019;235:1-3.

20 Rayner JE, Laino AM, Nufer KL, et al. Clinical perspective of 3D total body photography for early detection and screening of melanoma. Front Med 2018;5.

21 Coory M, Smithers M, Aitken J, et al. Urban-rural differences in survival from cutaneous melanoma in Queensland. Aust N Z J Public Health 2006;30:71-4.

22 Finnane A, Dallest K, Janda M, et al. Teledermatology for the diagnosis and management of skin cancer: a systematic review. JAMA Dermatol 2017:153:319-27.

23 Watts CG, Madronio CM, Morton RL, et al. Diagnosis and clinical management of melanoma patients at higher risk of a new primary melanoma: a population-based study in New South Wales, Australia. Australas J Dermatol 2017;58:278-85.
24 Random.org. True random number service, 1998. Available: https:// www.random.org

25 Gamer M, Lemon J. Fellows Puspendra Singh I. Irr: various coefficients of interrater reliability and agreement 2010.

26 Landis JR, Koch GG. The measurement of observer agreement for categorical data. Biometrics 1977;33:159-74.

27 Koh U, Janda M, Aitken JF, et al. 'Mind your moles' study: protocol of a prospective cohort study of melanocytic naevi. BMJ Open 2018;8:e025857.

28 Horsham C, Loescher LJ, Whiteman DC, et al. Consumer acceptance of patient-performed mobile teledermoscopy for the early detection of melanoma. Br J Dermatol 2016;175:1301-10.

29 Orruño E, Gagnon MP, Asua J, et al. Evaluation of teledermatology adoption by health-care professionals using a modified technology acceptance model. J Telemed Telecare 2011;17:303-7.

30 Olsen CM, Green AC, Neale RE, et al. Cohort profile: the QSkin sun and health study. Int J Epidemiol 2012;41:929-929i.

31 Pickard AS, Wilke CT, Lin H-W, et al. Health utilities using the EQ-5D in studies of cancer. Pharmacoeconomics 2007;25:365-84.

32 Osborne S. Influences on compliance with standard precautions among operating room nurses. Am J Infect Control 2003;31:415-23.

33 Aspinwall LG, Taber JM, Kohlmann W, et al. Perceived risk following melanoma genetic testing: a 2-year prospective study distinguishing subjective estimates from recall. $J$ Genet Couns 2014;23:421-37.

34 Chan A-W, Tetzlaff JM, Altman DG, et al. SPIRIT 2013 statement: defining standard protocol items for clinical trials. Ann Intern Med 2013;158:200-7.

35 Gilmore S. Melanoma screening: informing public health policy with quantitative modelling. PLoS One 2017;12:e0182349.

36 (AlHW) AloHaW. Analysis of cancer outcomes and screening behaviour for National cancer screening programs in Australia, 2018 Available: https://www.aihw.gov.au/getmedia/0c4daae9-d0c6-422aafbd-47a36c6c8697/aihw-can-115.pdf.aspx?inline=true 